\title{
Hermite Coherent States for Quadratic Refractive Index Optical Media
}

\author{
Zulema Gress and Sara Cruz y Cruz
}

\begin{abstract}
Ladder and shift operators are determined for the set of HermiteGaussian modes associated with an optical medium with quadratic refractive index profile. These operators allow to establish irreducible representations of the $s u(1,1)$ and $s u(2)$ algebras. Glauber coherent states, as well as $s u(1,1)$ and $s u(2)$ generalized coherent states, were constructed as solutions of differential equations admitting separation of variables. The dynamics of these coherent states along the optical axis is also evaluated.
\end{abstract}

Keywords Hermite-Gaussian modes - Ladder operators - Coherent states · Self-focusing media $\cdot$ Paraxial beams $\cdot$ Ermakov equation

\section{Introduction}

The problem of addressing the construction, analysis, and possible applications of coherent states has been a very important issue in quantum physics. Yet, the concept of coherent state has also been considered within the framework of classical optics in the context of the quantum mechanics-classical optics analogy [1-3]. This analogy, based on the formal equivalence between the paraxial Helmholtz equation and the time-dependent Schrödinger equation [4-6], enables, for instance, the use of operatorial methods for the description of light propagation phenomena $[7,8]$. In particular, diverse families of coherent states as linear combinations of

\footnotetext{
Z. Gress

Universidad Autónoma del Estado de Hidalgo, Ciudad del Conocimiento, Hidalgo, Mexico

S. Cruz y Cruz $(\bowtie)$

Instituto Politécnico Nacional, UPIITA, Ciudad de México, Mexico

e-mail:sgcruzc@ipn.mx 